\title{
EFECTOS DE PRODUCTOS DOPANTES EN FLUIDOS PARA PRUEBAS DE DESPLAZAMIENTO USANDO TOMOGRAFIA COMPUTARIZADA
}

Fabián Andrés Tapias Hernández ${ }^{1 *}$, Nicolás Santos Santos ${ }^{2}$, Edwar Hernando Herrera Otero ${ }^{3}$

*A quien debe dirigirse la correspondencia

\section{RESUMEN}

En Colombia se está innovando en el uso de la tomografía computarizada para el estudio de métodos de recobro mejorado que puedan ser aplicados en campos maduros, es decir, aquellos que han alcanzado el pico máximo de producción y han entrado en una etapa de declinación. La tomografía computarizada ha mostrado sus ventajas en la industria médica, entre otras. La técnica se basa en el uso de rayos X para obtener cortes o secciones reconstruidas digitalmente de fluidos, objetos o materiales.

La visualización de fluidos puede ser mejorada empleando productos de contraste o también conocidos como dopantes. En la industria de los hidrocarburos, dichos productos son usados en pruebas de desplazamiento con el fin de generar una diferenciación gráfica notable entre los fluidos inyectados en la roca. Su uso ha sido poco documentado, por lo cual, la presente investigación tiene como objetivo realizar un estudio inicial sobre el efecto que tendrán los dopantes en la visualización de imágenes y en la viscosidad de fluidos. Para esto, se llevó a cabo una búsqueda bibliográfica sobre el efecto en la visualización de las imágenes resultantes del proceso. Además, se elaboró y aplicó un diseño experimental para evaluar el efecto de este tipo productos en la viscosidad de 2 fluidos hidrocarburos y una salmuera. Lo anterior, apuntando a establecer bases conceptuales y experimentales que contribuyan a la curva de aprendizaje acerca del uso de esta técnica en la ingeniería de petróleos.

Palabras clave: Dopantes, Tomografía computarizada, Viscosidad.

\section{EFFECTS OF DOPANT PRODUCTS ON FLUIDS FOR CORE FLOODING TESTS USING COMPUTED TOMOGRAPHY.}

\begin{abstract}
Colombia is innovating in the use of computerized tomography for the study of enhanced oil recovery methods that could be applied on mature fields, i.e., those that have reached the maximum production and have entered in a declining stage. The computerized tomography has proven advantageous in the medical field and among others. The technique is based in the use of $\mathrm{X}$ rays to obtain digitally reconstructed cross sections from fluids, objects or materials.
\end{abstract}

The fluids visualization can be improved using contrast products, also known as dopants. In the petroleum industry, those products are used in displacement tests with the purpose of generating a remarkable contrast for the injected

1. Departamento Ingeniería de Petróleos, Facultad Ingeniería Mecánica. Universidad Estadual de Campinas. São Paulo. Brasil. Email: fabian.tapias@hotmail.com

2. Escuela Ingeniería de Petróleos. Universidad Industrial de Santander. Bucaramanga. Colombia. Email: nicolas@ uis.edu.co

3. Corporación Natfrac, Piedecuesta, Colombia. Email: Email: edwar.herrera@natfrac.com 
fluids in the rock. Its use has been poorly documented, whereby, the present research has the objective of developing an initial study on the effect that dopants will have in images visualization and fluids viscosity. For this purpose, a bibliographical review about the effect on images resulting from these processes was carried out. Moreover, an experimental design was elaborated to evaluate the effect of those products on viscosity for two petroleum and a brine sample. The above, aiming to establish conceptual and experimental bases that contribute to the learning curve about the use of this technique in the petroleum engineering.

Keywords: Dopants, Computerized tomography, viscosity.

\section{INTRODUCCIÓN}

La recuperación mejorada de hidrocarburos involucra la inyección de uno o más fluidos con el fin de desplazar fluidos presentes en el yacimiento buscando mejorar la eficiencia de barrido e incrementar la cantidad de petróleo que se extrae del yacimiento. Para investigar la efectividad de tales desplazamientos, en laboratorio se llevan a cabo pruebas inyección de fluidos, usando un medio poroso saturado con fluidos de interés tales como agua y petróleo. Esta práctica no es usada solamente como predicción del comportamiento de flujo en un yacimiento, también suele ser usada como herramienta base de calibración para modelos de simulación numérica.

Durante el desarrollo tecnológico de estas pruebas de laboratorio siempre ha sido difícil tener una vista detallada de los mecanismos de desplazamiento insitu debido a la naturaleza opaca del portamuestras y la distribución espacial de los fluidos desplazados. Sin embargo, la disponibilidad de técnicas de imágenes para la visualización tri-dimensional detallada de la muestra y visualización del movimiento de fluidos en el medio poroso durante el desplazamiento, ha sido reportada por (Wang, et al., 1984), (Hove, et al., 1987), (Vinegar \& Wellington, 1986), (Hunt \& Engler, 1987), (Withjack, 1988), (Withjack, 2003). Estos autores presentan los resultados de estudios hechos usando tomografía computarizada (CT) para observar el proceso de desplazamiento in-situ. Los resultados constituyen una nueva percepción dentro de los mecanismos de desplazamiento de fluidos en el medio poroso.

Los principios de la generación de imágenes a partir de rayos $\mathrm{X}$ han sido extensamente tratados por (Hounsfield, 1973), (Ledley, et al., 1974), (Payne \& McCullough, 1976), (Mackay, 1984). Básicamente, con la tomografía computarizada a partir de secciones transversales de un objeto tomadas en diferentes direcciones, se generan imágenes y se crea una reconstrucción del mismo. Lo anterior, basándose en la medición de atenuación de los rayos $\mathrm{X}$ al atravesar el objeto. Cuando el rayo $\mathrm{X}$ pasa a través de un material, algunos protones son absorbidos (Efecto fotoeléctrico) y algunos son dispersados (Efecto
Compton). El efecto fotoeléctrico esta principalmente relacionado con el número atómico del material y la energía del rayo (E, energía ionizante) mientras el efecto Compton está relacionado principalmente con la densidad del material (Payne \& McCullough, 1976). Estos procesos ocasionan que la cantidad de protones emitidos no sea igual a la cantidad de protones captados, efecto llamado atenuación del rayo X. El uso de CT en la ingeniería de petróleos se fundamenta en acompañar la realización de pruebas de desplazamiento con tomografía. Dicha práctica, requiere de un procesamiento remoto de la información digital obtenida en imágenes para llegar a correlacionar propiedades petrofísicas y perfiles de saturación a partir de los datos de atenuación. Teniendo en cuenta los principios fundamentales de la técnica, se hace necesario agregar productos que permitan diferenciar los fluidos dentro la roca, es decir, generar diferentes atenuaciones para cada fluido que satura el medio poroso. Estos productos son conocidos como dopantes o medios de contraste. La presente investigación abordó el estudio teórico experimental inicial sobre el efecto que tendrán dichos productos en la en la visualización de imágenes y en la viscosidad de fluidos.

\section{TOMOGRAFIA COMPUTARIZADA}

Según Wellington \& Vinegar, 1987, los tomógrafos han tenido un gran desarrollo desde 1972. La primera generación de escáner usaba un emisor de rayos $\mathrm{X}$ y un arreglo de detectores de señal. La segunda generación mejoró la calidad de la imagen mediante el uso de múltiples detectores en una configuración que rota y traslada a medida que avanza la prueba. Una notoria diferencia en velocidad durante la realización de pruebas fue presentada en los escáneres CT de tercera generación, estos usaban una configuración en la cual la fuente y los detectores rotan juntos alrededor del objeto. La cuarta generación de escáneres CT usa una fuente de rayos X que gira y bombardea la muestra con electrones mientras anillos de detectores de alta eficiencia captan la atenuación de los rayos. La cantidad básica de medida por cada pixel de la imagen CT es el coeficiente de atenuación linear del rayo X. Esta es definida por la ley de Beer: 


$$
\frac{I}{I_{o}}=\exp ^{(-\mu h)}
$$

Donde Io es la intensidad de incidencia del rayo X, I es la intensidad remanente después que los rayos $\mathrm{X}$ pasan a través de un espesor h. El coeficiente de atenuación linear $(\mu)$ depende de la densidad $(\rho)$ y el número atómico $(\mathrm{Z})$ del material, como se muestra a continuación:

$$
\mu=\rho\left(a+\frac{b Z^{3,8}}{E^{3,2}}\right)
$$

Donde $a$ es una aproximación del coeficiente de energía independiente llamado coeficiente Klein-Nishina y $b$ es una constante (Evans, 1955), (Peters \& Hardham, 1990). El primer término de la ecuación 2, representa el efecto Compton, el cual es predominante a energías de rayo-X por encima de $100 \mathrm{kV}$. El segundo término hace referencia al efecto fotoeléctrico, siendo más importante a energías de rayo-X por debajo de $100 \mathrm{kV}$. Operar el scanner CT a dos energías de rayo diferentes es conocido como metodología de Energía dual (Dual Energy); es logrado por el cambio de voltaje en la fuente emisora de rayos $\mathrm{X}$.

Los datos resultantes del CT son normalmente presentados en una escala internacional de unidades denominada Hounsfield $(\mathrm{H})$ definida por el aire a -1000 $[\mathrm{H}]$ y el agua a $0[\mathrm{H}]$. Cada unidad Hounsfield representa un $0,1 \%$ de cambio en la densidad del material y es también conocido como un número CT. (Wellington \& Vinegar, 1987).

\section{USO DE PRODUCTOS DOPANTES}

El espacio poroso de las rocas usualmente contiene un volumen de agua mínimo e inmóvil conocido como saturación de agua connata (Swc). Adicionalmente, las rocas contienen una saturación de petróleo (So) y dependiendo del tipo de yacimiento pueden contener gas (Sg) y una cantidad de agua móvil (Sw). El agua presente en el yacimiento está compuesta por diferentes sales, pudiéndose denominar también salmuera.

Los dopantes son fluidos de alto número atómico usados para la determinación de saturación bifásica, i.e, aguapetróleo, o trifásica, i.e, agua, petróleo, gas; durante la caracterización dinámica de fluidos en medios porosos usando tomografía computarizada.
Cuando se tienen diferentes fluidos en el medio poroso con diferentes números atómicos, el efecto fotoeléctrico obtenido durante la prueba $\mathrm{CT}$, se denomina número atómico efectivo, Ze, (Wellington \& Vinegar, 1987) y corresponde a:

$$
Z_{e}=\left(\sum P_{i} Z_{i}^{3,8}\right)^{\frac{1}{3,8}}
$$

Donde, $P_{i}$ es la razón de electrones de cada elemento respecto al total de electrones que compone una especie. Por ejemplo, el número atómico efectivo del metano (CH4) es:

$$
\begin{gathered}
Z_{e}=\left(\frac{1}{10} * 6^{3,8}\right)^{\frac{1}{3,8}}+\left(\frac{4}{10} * 1^{3,8}\right)^{\frac{1}{3,8}} \\
Z_{e}=3,27336+0,78574=4,05910
\end{gathered}
$$

En el ejemplo, se puede evidenciar que el Ze medido en la tomografía computarizada es fuertemente influenciado por el elemento de mayor número atómico. La relación directa entre el efecto fotoeléctrico y el número atómico es utilizada para mejorar la diferencia de atenuación entre los diferentes fluidos que saturan un medio poroso.

A escala de laboratorio se debe garantizar que la saturación de fluidos en las muestras de roca sea aproximada a la del yacimiento. El conjunto de procedimientos petrofísicos llevados a cabo para garantizar estas condiciones se denomina restauración de la muestra de roca.

Una vez restaurada la muestra de roca, se tendrá una distribución de fluidos en el medio poroso (p.ej, aguapetróleo) con un coeficiente de atenuación másico total dado por:

$$
\frac{\mu_{T}}{\rho_{\mathrm{T}}}=\sum_{i=1}^{n}\left(\frac{\mu_{i}}{\rho_{i}}\right) f_{i}
$$

Donde $\mu_{i}=$ Es el coeficiente de atenuación de cada componente del fluido que satura completamente la muestra de roca, fi y $\rho_{i}$ son la fracción en peso y densidad de cada componente del fluido, respectivamente. Multiplicando la ecuación 2 por $\rho_{T}$ se tiene:

$$
\mu_{T}=\sum_{i=1}^{n} \mu_{i}\left(\frac{V_{i}}{V_{T}}\right)
$$


Para simplificar la ecuación 5, se denomina $S_{i}=\left(\frac{V_{i}}{V_{T}}\right)$ como la fracción volumétrica de determinado componente i. Por lo tanto, la ecuación 5 puede ser expresada como:

$$
\mu_{T}=\sum_{i=1}^{n} \mu_{i} S_{i}
$$

$\mathrm{Si}$ los componentes son inmiscibles, Si representa la saturación existente de cada una de las fases. Si los fluidos son miscibles y los volúmenes aditivos, la ecuación 6 puede ser utilizada teniendo presente que $\mathrm{Si}$ no es la fase saturante, basados en lo anterior, el espacio poroso se encuentra ocupado por una distribución de componentes de todas las fases. Por ejemplo, en un flujo inmiscible de $\mathrm{CO} 2$, el dióxido de carbono puede formar una única fase con el petróleo y también puede disolverse en el agua de formación presente hasta cierto grado. La interpretación de $S_{\mathrm{CO}^{2}}$ a partir de los datos CT no es la saturación ocasionada por el gas libre, sin embargo, puede ser considerada como la fracción volumétrica ocupada por el $\mathrm{CO} 2$ dentro de cada una de las fases. Esto siempre y cuando se consideren volúmenes aditivos (1 cc de $\mathrm{CO} 2$ mezclado con $1 \mathrm{cc}$ de petróleo resulta en $2 \mathrm{cc}$ de mezcla). (Vinegar \& Wellington, 1986).

Para la estimación de saturaciones bifásicas la energía ionizante tendrá un coeficiente de atenuación diferente tanto para el agua presente en el yacimiento como para el petróleo. Es decir,

$$
\mu=\mu_{w} S_{w}+\mu_{o} S_{o}
$$

Asumiendo que el medio poroso está ocupado solamente por agua y petróleo, se tiene:

$$
S_{w}+S_{o}=1
$$

Despejando Sw de la ecuación 8 y reemplazando en la ecuación 7:

$$
S_{o}=\frac{\mu-\mu_{w}}{\mu_{o}-\mu_{w}}
$$

En el caso de saturaciones trifásicas se deben realizar al menos dos mediciones, cada una a diferente nivel de energía (E1 y E2), lo cual origina un sistema de 3 ecuaciones con 3 incógnitas:

$$
\begin{gathered}
\mu_{1}=\mu_{w 1} S_{w}+\mu_{o 1} S_{o}+\mu_{g 1} S_{g} \\
\mu_{2}=\mu_{w 2} S_{w}+\mu_{o 2} S_{o}+\mu_{g 2} S_{g} \\
S_{o}+S_{w}+S_{g}=1
\end{gathered}
$$

Sustituyendo $\mu_{i}-\mu_{g}=\mu_{i}$ y despejando cada termino de saturación en función del coeficiente de atenuación de cada componente del fluido, se tiene:

$$
\begin{gathered}
S_{w}=\frac{\mu_{02} \mu_{1}-\mu_{01} \mu_{2}}{\mu_{02} \mu_{w 1}-\mu_{01} \mu_{w 2}} \\
S_{w}=\frac{-\mu_{w 2} \mu_{1}-\mu_{w 1} \mu_{2}}{\mu_{02} \mu_{w 1}-\mu_{01} \mu_{w 2}} \\
S_{g}=1-S_{w}-S_{o}
\end{gathered}
$$

Las ecuaciones sean para un sistema bifásico o trifásico son dependientes del coeficiente de atenuación másico por ende se debe garantizar una diferenciación entre el efecto Compton y el efecto fotoeléctrico; que puede ser efectuada con el uso de dopantes generando una potencialización del efecto fotoeléctrico y de esta manera una diferencia notable de contraste en las imágenes obtenidas a alta y baja energía. En las figura 1, se muestra el incremento del coeficiente de atenuación del petróleo en un sistema trifásico usando 1 dopante.

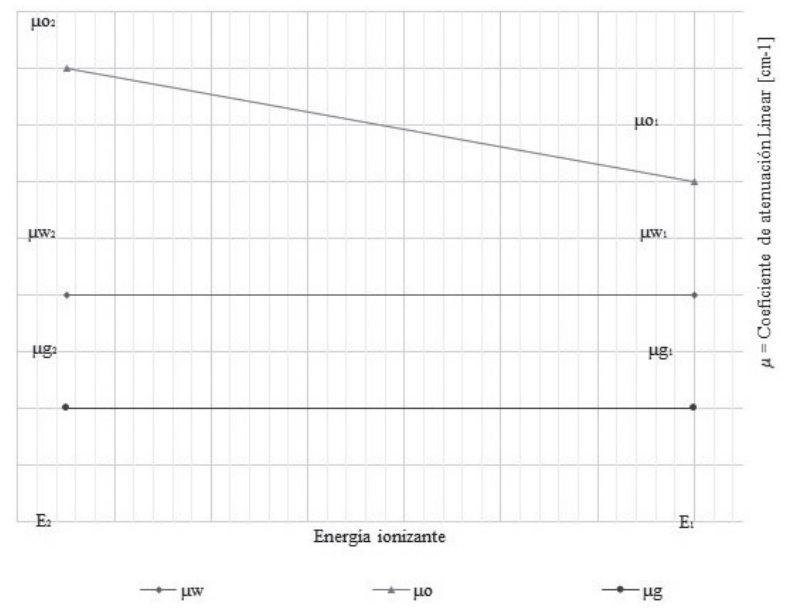

Figura 1. Atenuación linear VS Energía óptima para 1 Dopante.

Fuente: (Vinegar \& Wellington, 1986), modificado por los autores.

\section{SELECCIÓN DE NIVEL DE ENERGÍA Y DOPANTE APROPIADO}

Debido a que los números Hounsfield del petróleo y el agua son similares es necesario agregar al agua, al petróleo o a ambos fluidos materiales con mayor coeficiente de absorción. El usar uno o dos dopantes depende del diseño experimental de las pruebas de desplazamiento. Es decir, si se va desplazar un único fluido a través de una muestra restaurada a condiciones 
de yacimiento se recomienda usar un solo dopante. En el caso, de querer estudiar el incremento de recobro por inyección de agua, lo recomendado es usar el producto en el agua a inyectar ya que el cambio en la viscosidad del fluido tiende a ser poco relevante (Wellington \& Vinegar, 1987), además el consumo energético se reduce; solo es necesario utilizar una energía que maximice la absorción de rayos $\mathrm{X}$ en el fluido dopado. Con lo cual, la sensibilidad en la visualización del fluido en particular se ve notoriamente mejorada.

El uso de dos dopantes es recomendado cuando se tiene la necesidad de diferenciar más de dos fluidos, sea en un método de recuperación miscible o un método de recobro químico como surfactante-polímero (SP) o álcali-surfactante-polímero (ASP). (Vinegar \& Wellington, 1986) Recomiendan tener en cuenta los siguientes principios para la escogencia de dopantes y energía ionizante a utilizar en un proceso de inyección de fluidos en el medio poroso con CT:

1) La radiación a determinada energía (i.e, E1>100 $\mathrm{Kv}$ ) deber ser absorbida inicialmente por el agua y la radiación a otra energía (i.e, E2 $<100 \mathrm{Kv}$ ) absorbida principalmente por el petróleo. Matemáticamente puede expresarse de la siguiente forma:

$$
\begin{aligned}
& \frac{\left(\mu_{w 1}^{\prime}\right)}{\left(\mu_{o 1}^{\prime}\right)} \rightarrow \text { Maximizado } \\
& \frac{\left(\mu_{w 2}^{\prime}\right)}{\left(\mu_{o 2}^{\prime}\right)} \rightarrow \text { Minimizado }
\end{aligned}
$$

Donde cada subíndice indica la energía a la que fue medido el coeficiente de atenuación. En las figura 2, se muestra que al usar una E1, el coeficiente de atenuación del agua es maximizado y el del petróleo minimizado, mientras que con una E2, se maximiza el coeficiente de atenuación del petróleo y se minimiza el del agua.

2) El cuadrado de los coeficientes de absorción debe ser igual a ambas energías:

$$
\left(\mu_{w 1}^{\prime}\right)^{2}+\left(\mu_{o 1}^{\prime}\right)^{2}=\left(\mu_{w 2}^{\prime}\right)^{2}+\left(\mu_{o 2}^{\prime}\right)^{2}
$$

3) Al utilizar el método de energía dual usar las aproximaciones de Energía de absorción o "K-absorción" (Storm \& Israel, 1970) de elementos pesados en la región fotoeléctrica, procurando una diferencia notable entre el valor de este parámetro para cada fluido. Por ejemplo, un par de dopantes apropiado seria el yododecano para petróleo (Petróleo yodado) y tungstato de sodio para el agua. La K-absorción se expresa en Kev y hace referencia a la unidad de medida de la energía de los fotones del rayo $X$.

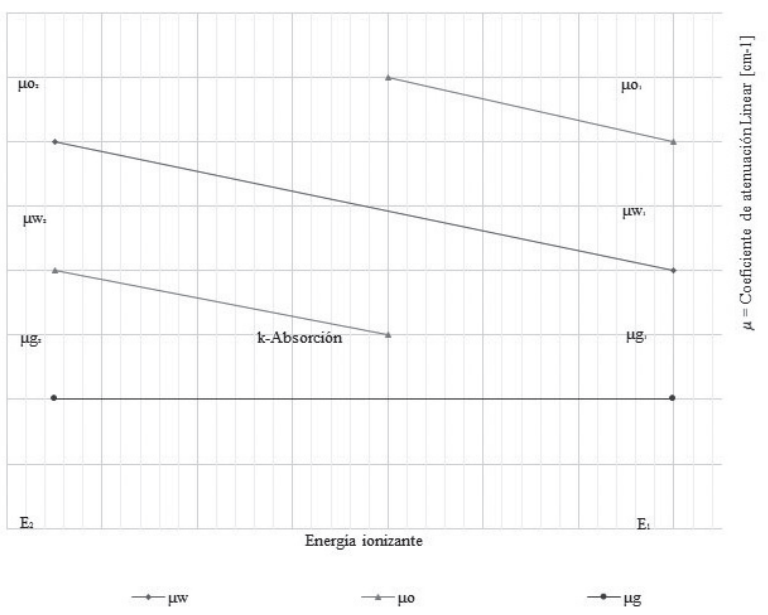

Figura 2. Atenuación linear VS Energía óptima para 2 Dopantes.

Fuente: (Vinegar \& Wellington, 1986), modificado por el autor.

En las tablas 1-3, se muestra la "K-absorción" de algunos productos dopantes.

Tabla 1. Productos dopantes para salmuera

\begin{tabular}{|cc}
\hline Nombre & $\mathrm{K}(\mathrm{KeV})$ \\
\hline Bromuro de Sodio & 13,2 \\
\hline Molibdato de Sodio & 20 \\
\hline Yoduro de sodio & 33,2 \\
\hline Tungstato de sodio & 69,5 \\
\hline Fluoruro de talio & 85,5 \\
\hline Nitrato de Plomo & 88 \\
\hline
\end{tabular}

Fuente: (Vinegar \& Wellington, 1986), modificado por los autores.

Tabla 2. Productos dopantes para petróleo

\begin{tabular}{|cc|}
\hline Nombre & K (KeV) \\
\hline Petróleo Bromado & 13,2 \\
\hline Petróleo Yodado & 33,2 \\
\hline Tetraetil-plomo & 88 \\
\hline Tetrametil-plomo & 88 \\
\hline
\end{tabular}

Fuente: (Vinegar \& Wellington, 1986), modificado por los autores. 
Tabla 3. Productos dopantes para gas

\begin{tabular}{cc} 
Nombre & K (KeV) \\
\hline Kriptón & 14,3 \\
\hline Xenón & 34,6 \\
\hline
\end{tabular}

Fuente: (Vinegar \& Wellington, 1986), modificado por los autores.

4) Comparar el comportamiento de los fluidos con y sin dopante en pruebas de comportamiento de fases, miscibilidad y tensión interfacial, buscando el producto que minimice las diferencias de comportamiento respecto a los fluidos originales.

\section{ARTEFACTOS DE IMAGEN}

Los artefactos son características de la imagen que son producidas por el procesamiento de la misma y no están presentes en el objeto original (Peters \& Hardham, 1990). El artefacto más conocido es el Beam Hardening o endurecimiento del rayo que existe debido a que los fotones que componen el rayo $\mathrm{X}$, son más fácilmente atenuados a una baja energía $(\mathrm{E}<100 \mathrm{kV})$. Este artefacto se caracteriza por generar altos coeficientes de atenuación en la periferia del objeto y bajos coeficientes de atenuación en proximidades del centro del objeto. Los tomógrafos médicos comerciales tienen una serie de correcciones para dicho efecto. La utilización de estas correcciones en la ingeniería de petróleos se ve limitada dependiendo del material del portamuestras, los tipos de fluidos y los productos dopantes escogidos. Es por esto que una escogencia inadecuada de los dopantes y sus concentraciones, puede minimizar los beneficios que ofrece el CT en pruebas de desplazamiento de fluidos en el medio poroso. A continuación se muestra el efecto generado por los dopantes durante el escáner de una muestra de roca a dos energías.

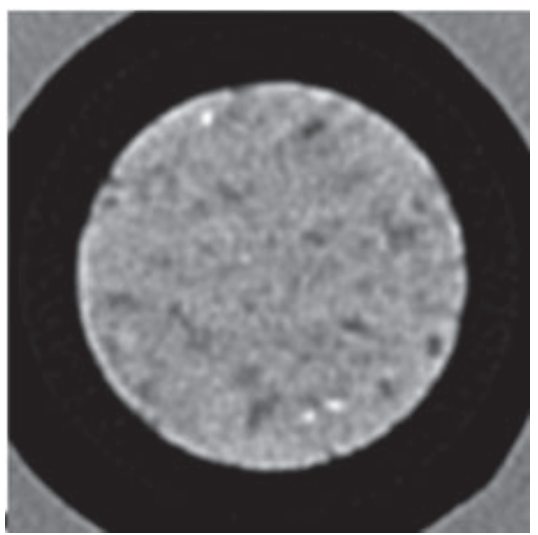

Figura 3.a. Sección de la muestra completamente saturada con agua de formación a $130 \mathrm{kV}$.

Fuente: (Gonçalves Machado, 2013)
En la figura 3.a, la visualización es buena, permite ver materiales de mayor densidad en la muestra de roca (color blanco), presenta una buena diferenciación del portamuestras y la roca, no se evidencia una disminución de los números Hounsfield hacia el centro de la imagen (no hay presencia de artefactos).

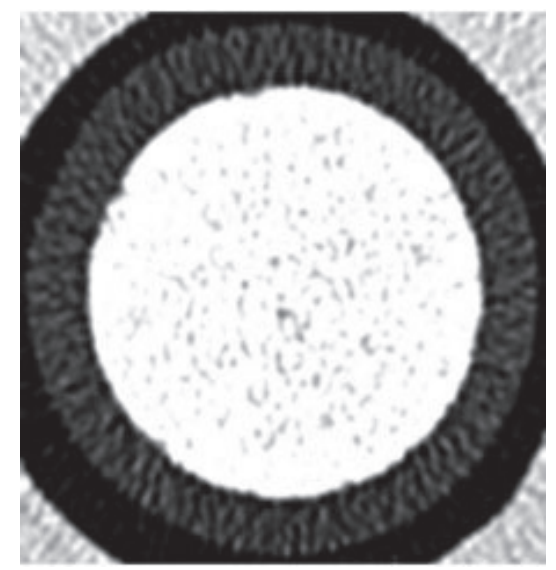

Figura 3.b. Sección de la muestra completamente saturada con agua de formación a $80 \mathrm{kV}$.

Fuente: (Gonçalves Machado, 2013)

La figura 3.b, evidencia la presencia del endurecimiento del rayo ocasionado por una equivoca escogencia de la concentración de dopante usado; generando pérdida de calidad en la visualización y dificultando la diferenciación gráfica de fluidos en el medio poroso.

\section{EFECTOS DE LOS DOPANTES SOBRE LOS FLUIDOS}

Gonçalves Machado, 2013, efectuó mediciones reológicas y de densidad al aceite mineral EMCA de $31^{\circ}$ API con $10 \%$ en peso (wt) de yododecano y una salmuera de 200000 partes por millón (ppm) con una composición de $0,5 \%$ wt de yodato de sodio y $0,5 \%$ wt de cloruro de sodio. Las medidas fueron efectuadas antes de iniciar el proceso de desplazamiento y al finalizar con los fluidos obtenidos. Las variaciones resultantes no fueron significativas por lo tanto se afirmó que no hubo incidencia reológica de los productos dopantes sobre los fluidos de interés.

En 1986, Vinegar \& Wellington estudiaron la influencia de productos dopantes (Hexadecano y Yododecano) en el Soltrol 130, aceite refinado alifático con un reducido número de carbonos entre 10 y 13 , ampliamente usado en la ingeniería de petróleos para pruebas de desplazamiento de fluidos en medios porosos. En la Figura 4, se muestra el comportamiento de la viscosidad 
del Soltrol 130 respecto a los productos dopantes en función de la temperatura.

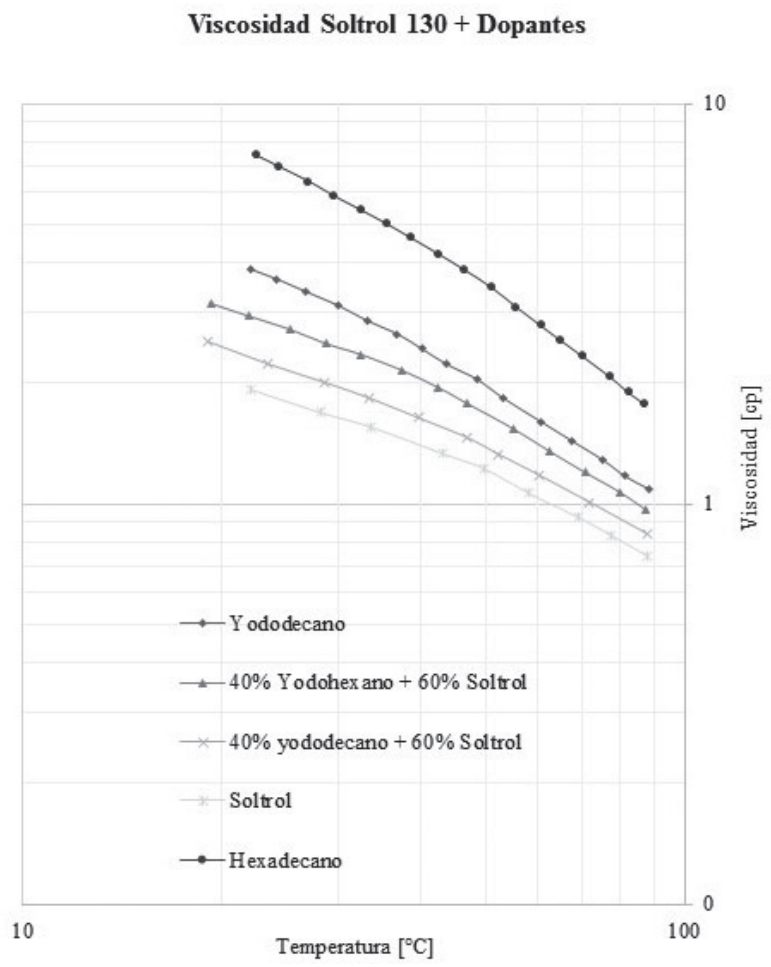

Figura 4. Viscosidad de la mezcla yododecano/soltrol hexadecano/soltrol en función de la temperatura.

Fuente: (Vinegar \& Wellington, 1986), modificada por los autores.

El dopante escogido fue el yododecano; los autores atribuyen la reducida variación del comportamiento de la viscosidad a la pequeña diferencia en la composición molecular entre el Soltrol 130 y el yododecano. Este último, solo difiere del aceite mineral en un átomo de hidrogeno que fue reemplazado con Iodino.

La teoría de Buckely-Leverett para tres fases fue verificada por (Siddiqui, et al., 1996), apoyándose en la tomografía computarizada y con el uso de productos dopantes, reportando buenos resultados. Un buen ajuste de esta teoría no podría haberse llevado a cabo si las propiedades de los fluidos inyectados en el medio poroso hubiesen mudado drásticamente con el uso de los dopantes.

\section{DESARROLLO EXPERIMENTAL}

Se realizó un planteamiento experimental para evaluar el efecto de tres productos dopantes diferentes en la viscosidad de 2 fluidos hidrocarburos y una salmuera. Se decidió evaluar esta propiedad en particular debido a los estudios realizados por (Gonçalves Machado, 2013) (Vinegar \& Wellington, 1986) (Wellington \& Vinegar, 1987). Además de esto, (Sheng, 2013), (Dake, 2005) resaltan la importancia de la viscosidad en procesos de recobro mejorado.

\subsection{SELECCIÓN DE FLUIDOS DE ESTUDIO Y PRODUCTOS DOPANTES.}

La selección de los fluidos se basó en los intereses del proyecto de investigación "Técnicas avanzadas de imágenes en medios porosos para la caracterización no intrusiva de roca e incremento del factor de recobro en campos de crudo pesado y campos maduros de crudos convencionales". Los fluidos escogidos fueron petróleo de campo Tulipán, campo Jilguero 1 y salmuera del campo San Francisco.

Los productos dopantes escogidos fueron Yodato de potasio (KIO3), nitrato de plomo ( $\mathrm{Pb}(\mathrm{NO} 3) 2)$ y yodo. Se fundamentó en los criterios propuestos por (Vinegar \& Wellington, 1986), Tablas 1 y 2.

\subsection{PROPIEDADES DE LOS FLUIDOS}

En la tabla 4 se muestra la gravedad API de cada hidrocarburo determinada en el laboratorio de análisis petrofísicos de la Universidad Industrial de Santander. La densidad fue determinada teóricamente a través del concepto de gravedad API (Ecuación 14) y tomando como referencia la densidad del agua a $60\left[{ }^{\circ} \mathrm{C}\right]$.

$$
\begin{gathered}
{ }^{\circ} A P I=\frac{141,5}{\Upsilon}-131,5 \\
Y=\frac{\text { Densidad Fluido }}{\text { Densidad de Referencia }}
\end{gathered}
$$


Tabla 4 Propiedades de los hidrocarburos

\begin{tabular}{ccc} 
& Jilguero 1 & Tulipán \\
\hline Gravedad API & 25,2 & 34,5 \\
Densidad $(\mathrm{g} / \mathrm{cc})$ & 0,8878 & 0,8380 \\
\hline
\end{tabular}

La composición de la salmuera se muestra en la tabla 5 . La densidad de la salmuera del campo San Francisco es $1,0035[\mathrm{~g} / \mathrm{cc}]$.

Tabla 5. Composición de la Salmuera del Campo San Francisco

\begin{tabular}{lc}
\multicolumn{1}{c}{ Tipo de Sal } & Concentración [ppm] \\
\hline Cloruro de Sodio & 5493,207 \\
\hline Cloruro de Potasio & 149,615 \\
Cloruro de Calcio Dihidratado & 1664,723 \\
Cloruro de Magnesio Hexahidratado & 496,088 \\
\hline
\end{tabular}

Fuente: Proyecto de investigación Técnicas avanzadas de imágenes.

\subsection{DISEÑO EXPERIMENTAL.}

Para evaluar la viscosidad de cada fluido con y $\sin$ dopantes se usó un viscosímetro Brookfield DV2T de cilindros concéntricos, con una dispersión de resultados tolerable de $\pm 5 \%$. En la tabla 6 , se muestran las pruebas propuestas.

Tabla 6. Pruebas de viscosidad

\begin{tabular}{l}
\multicolumn{1}{c}{ MUESTRA } \\
Salmuera (SF) \\
SF + Yodato de potasio (YDT) \\
SF + Nitrato de Plomo (NTP) \\
SF + Yodo \\
Petróleo Campo Tulipán (Tul) \\
Tul + YDT \\
Tul + Yodo \\
Petróleo Campo Jilguero (Jil) \\
Jil + YDT \\
Jil + Yodo \\
\hline
\end{tabular}

Las pruebas fueron llevadas a cabo a $60\left[{ }^{\circ} \mathrm{C}\right]$, con dos repeticiones por muestra y siguiendo el procedimiento establecido por el laboratorio de análisis petrofísicos de la UIS.

\subsection{PREPARACIÓN DE MUESTRAS}

Se emplearon tubos de ensayo de 18 milímetros $(\mathrm{mm})$ de diámetro. Un volumen de fluido de 10 mililitros (ml) por tubo y una concentración de dopante de
$40 \%$ wt. Concentración máxima de dopante sugerida por (Vinegar \& Wellington, 1986). A continuación se describe el proceso efectuado:

Preparación de muestras de Salmuera:

1. Preparación de Salmuera sintética del Campo San Francisco.

2. Lavado del interior del tubo de ensayo con Salmuera, proceso denominado purga del recipiente.

3. Preparación de $10 \mathrm{ml}$ de solución con una concentración de $40 \%$ wt de cada dopante.

4. Sellado del tubo de ensayo.

5. Calentamiento de muestras durante 5 minutos a $60\left[{ }^{\circ} \mathrm{C}\right]$.

6. Agitación manual de cada muestra durante 5 minutos.

7. Verificación visual de disolución total de los productos dopantes.

8. En caso de tener una disolución parcial, repetir los ítems 5, 6 y 7 hasta obtener una disolución total de los productos.

9. Almacenamiento de las muestras por 24 horas para observar si se presenta alguna precipitación de los productos.

Preparación de muestras de hidrocarburos:

1. Preparación de $10 \mathrm{ml}$ de solución con una concentración de $40 \%$ wt de cada dopante.

2. Sellado del tubo de ensayo.

3. Calentamiento de muestras durante 5 minutos a $60\left[{ }^{\circ} \mathrm{C}\right]$.

4. Agitación manual de cada muestra durante 10 minutos.

5. Verificación visual de disolución total de los productos dopantes apoyada de luz led.

6. En caso de tener una disolución parcial, repetir los ítems 3, 4 y 5 hasta obtener una disolución total de los productos.

7. Almacenamiento de las muestras por 24 horas para observar si se presenta alguna precipitación de los productos.

Las soluciones de Salmuera con yodato de potasio y nitrato de plomo, presentaron una coloración traslucida. (Figura 5).

La solución de Salmuera con yodo presento una coloración naranja, reacción usual del yodo al ser disuelto en un disolvente polar. (Figura 6). 

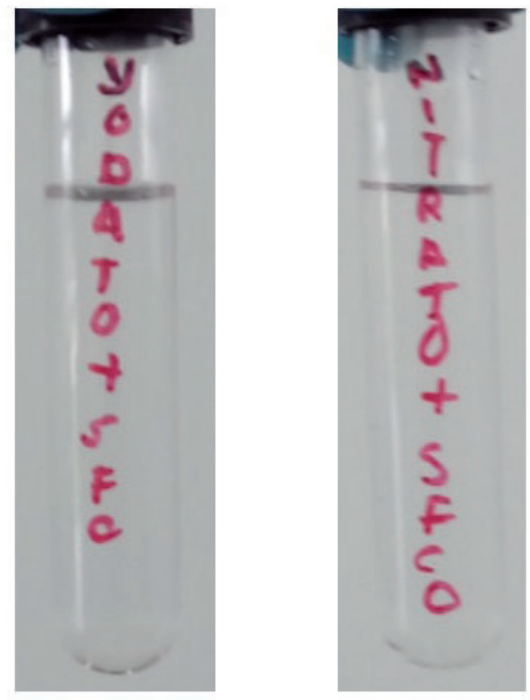

Figura 5. Soluciones de Salmuera con yodato de potasio y nitrato de plomo

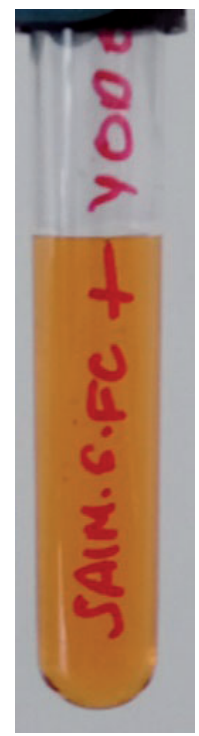

Figura 6. Soluciones de Salmuera con yodo

Los ítems 5, 6, 7 del proceso de preparación de muestras de salmuera fueron realizados en 5 ocasiones para la muestra de Salmuera con yodo, sin tener una disolución total del yodo. Por lo cual, se procedió a realizar el filtrado de la solución y pesaje del material no diluido. Luego, la solución fue puesta en nuevo tubo de ensayo garantizando la existencia de un volumen de $10 \mathrm{ml}$.

Finalmente, se calculó la nueva concentración de la solución de salmuera con yodo, siendo aproximadamente $36,5 \%$ wt.

\section{ANALISIS DE RESULADOS}

\subsection{PETRÓleo dE TULIPÁN}

En la figura 7, se muestran los resultados de las pruebas de viscosidad efectuadas para el petróleo del campo Tulipán. Las líneas punteadas corresponden a las repeticiones de cada solución

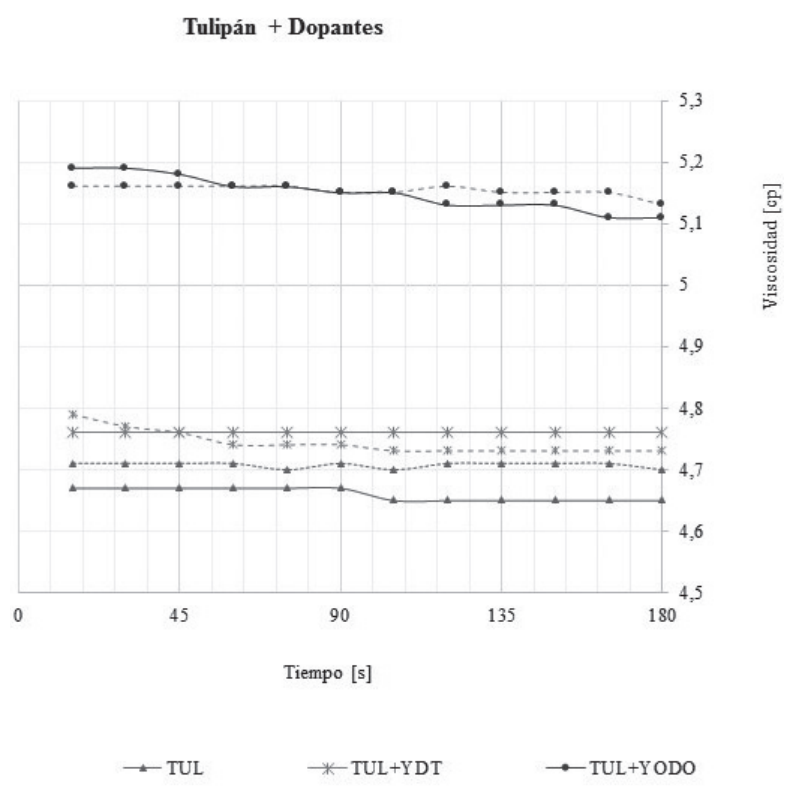

Figura 7. Comportamiento de la viscosidad del petróleo de campo Tulipán

Este hidrocarburo perdió su movilidad a temperatura de laboratorio, infiriendo un punto de fluidez cercano a [20 $\pm 5^{\circ} \mathrm{C}$. Comportamiento usual de hidrocarburos con alto contenido parafínico.

La dispersión de datos entre las repeticiones no excede el $1,1 \%$ para cada muestra. Se puede observar que los productos dopantes generan un un incremento en la viscosidad de este fluido. El yodo incrementó 0,6 [cp] la viscosidad, mientras que el incremento generado por el $\mathrm{KIO}_{3}$ fue de 0,2 [cp].

La variaciones de viscosidad en hidrocarburos livianos no son fuertemente significativas tal y como lo plantearon (Wellington \& Vinegar, 1987) (Vinegar \& Wellington, 1986) al experimentar con Soltrol 130 y (Gonçalves Machado, 2013) con aceite mineral. La similitud entre la composición molecular del fluido y el producto dopante, constituye un criterio de selección del producto a usar. 


\subsection{PETRÓleo de JILGUERO 1}

La figura 8, muestra el comportamiento de la viscosidad del petróleo del campo Jilguero La dispersión de datos máxima entre las repeticiones es del 1,8\% para cada muestra.

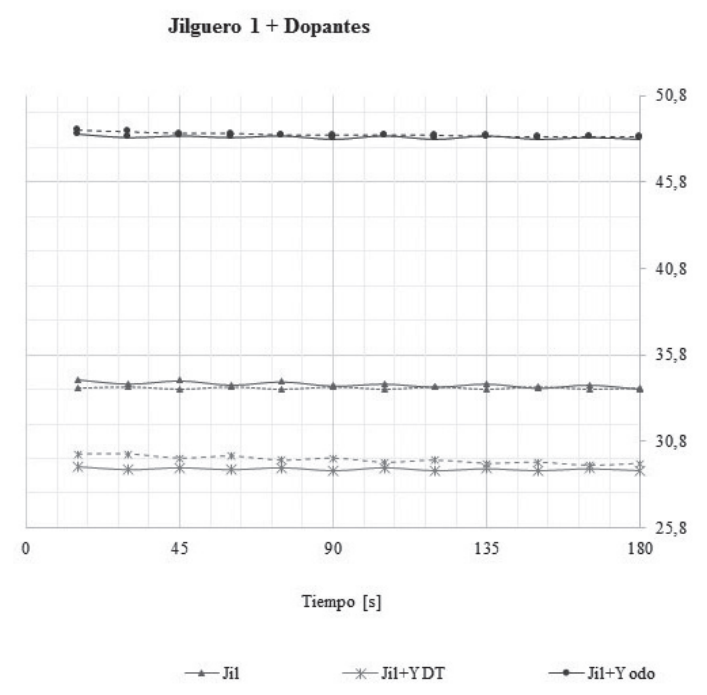

Figura 8. Comportamiento de la viscosidad de campo Jilguero 1

Los productos dopantes generan cambios importantes en la viscosidad de este hidrocarburo. $\mathrm{El}^{\mathrm{KIO}_{3}}$ disminuye la viscosidad 4,4 [cp.], mientras que el yodo produce un incremento de 14,3 [cp.].

(Cuñado , 2012), afirma que este hidrocarburo tiene una considerable fracción pesada, por tanto, la estructura molecular del fluido y los dopantes tiende a presentar grandes diferencias. Se piensa que la disolución del $\mathrm{KIO}_{3}$, en un compuesto apolar como el hidrocarburo tiende a romper enlaces y por lo tanto a descomponer las largas y complejas cadenas de carbonos. Por otro lado, el agregar un compuesto puro y de alta concentración por unidad de masa como el yodo tiende a fortalecer los enlaces entre las moléculas, favorecer el efecto dipolo dipolo e impactar negativamente la viscosidad.

Este último planteamiento, precisa de estudios posteriores en miras a un fundamento más robusto del mismo.

\subsection{SAlMUERA CAMPO SAN FRANCISCO}

El comportamiento de la viscosidad de la salmuera sintética del campo San Francisco es mostrado en la Figura 9. La dispersión de datos máxima entre las repeticiones es del $4,5 \%$.

El Pb $\left(\mathrm{NO}_{3}\right)_{2}$ tiende a aumentar la viscosidad mientras que los productos con yodo tienden a disminuirla. Los cambios generados por los productos dopantes en la salmuera no son muy significativos. Este comportamiento es consistente con el reportado por (Gonçalves Machado, 2013) y (Vinegar \& Wellington, 1986). Estos últimos, sugieren los productos dopantes ean agregados a la salmuera preferiblemente debido a que en proceso de recuperación mejorada las propiedades reológicas de los hidrocarburos pueden ser consideradas variables criticas del proceso de desplazamiento de fluidos en el medio poroso.

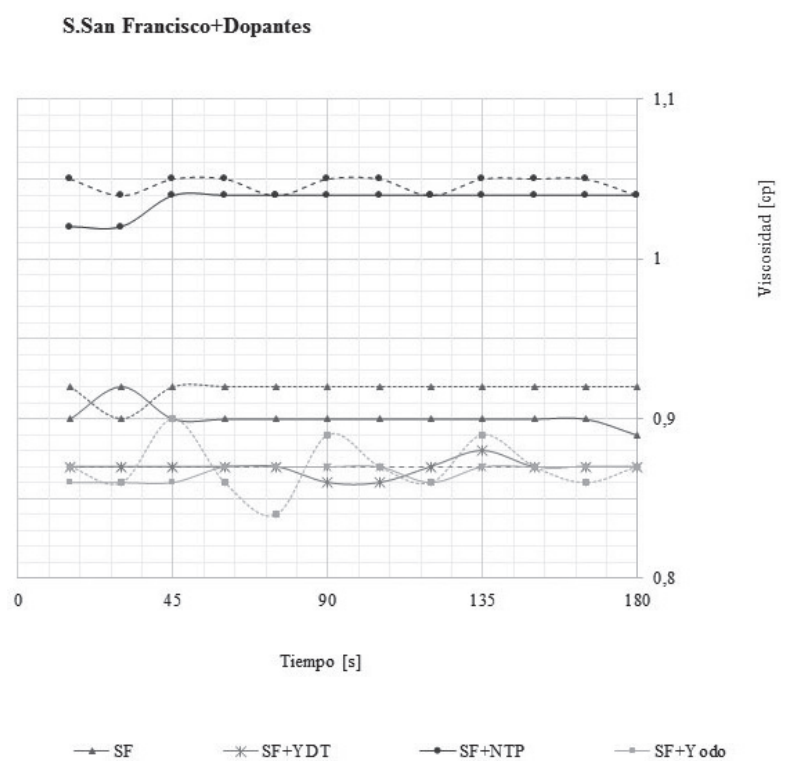

Figura 9. Comportamiento de la viscosidad de la Salmuera de Campo San Francisco

Se observó que la coloración naranja de la solución de salmuera con yodo desapareció durante la realización de las pruebas (figura 10). Se cree, el yodo tuvo una rápida oxidación al entrar en contacto continuo con el oxígeno y la luz. 
Se vislumbra la necesidad de realizar estudios posteriores que fundamenten el planteamiento anterior. El comportamiento de la prueba se vio influenciado por este fenómeno, la línea punteada de SF+Yodo en la figura 9, perdió completamente la proximidad hacia un comportamiento lineal, el cual es característico de fluidos newtonianos como el agua.

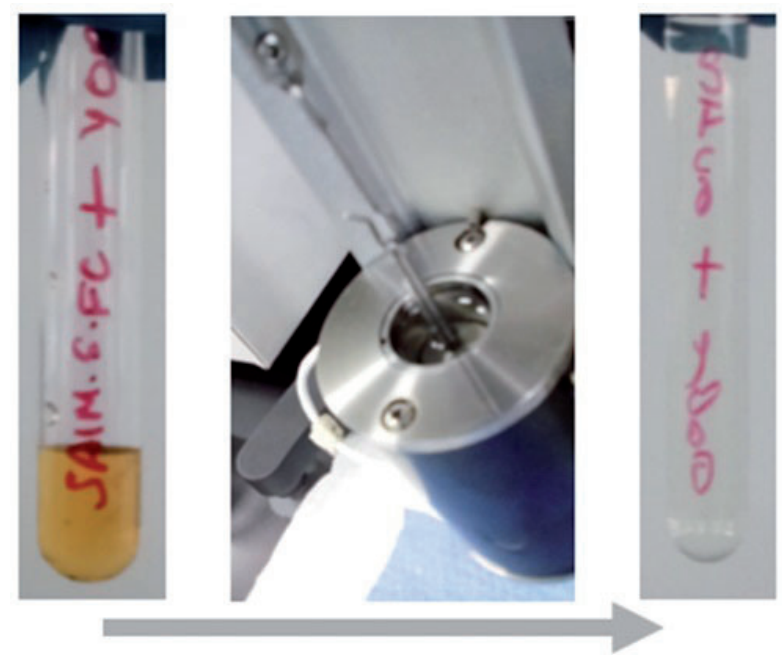

Figura 10. Cambio de coloración de la solución compuesta por Salmuera del Campo y yodo durante las pruebas de viscosidad.

\section{CONCLUSIONES}

Una incorrecta selección del producto dopante o la concentración a usar genera artefactos en las imágenes resultantes de la tomografía computarizada.

Las variaciones de viscosidad en fluidos hidrocarburos son poco significativas cuando se tiene una similitud entre la composición molecular del fluido y el producto dopante.

La escogencia de los productos dopantes tiene una relación directa con el número atómico de cada producto y por tanto con las energías en que se realiza el proceso de escaneo CT.

Los productos dopantes deben ser aplicados preferiblemente a la Salmuera, ya que la variación de viscosidad es mínima.

Determinar la concentración idónea de producto dopante a usar en un fluido hidrocarburo requiere de pruebas de comportamiento de fases, miscibilidad y tensión interfacial.
En procesos de energía dual la identificación de fluidos en el medio poroso es mejorada a medida que la diferencia de K-absorción de los productos dopantes es mayor

\section{RECOMENDACIONES}

El producto dopante seleccionado debe incluirse dentro de los cálculos de concentración de la salmuera sintética.

El tiempo entre la preparación de soluciones con yodo y la realización de pruebas debe ser mínimo.

Usar un medio inerte durante la manipulación del yodo para evitar la reacción con el oxígeno.

Utilizar tubos de ensayo de ámbar para prevenir la reacción del yodo con la luz y de esta manera reducir la rápida oxidación.

Usar agitadores mecánicos o magnéticos a fin de garantizar el mismo proceso de mezclado para todas las muestras.

\section{AGRADECIMIENTOS}

Con gran complacencia los autores del presente trabajo exponen sus más profundos agradecimientos al grupo de investigación en minerales, biohidrometalúrgia y medio ambiente (GIMBA) por los productos suministrados, al laboratorio de análisis petrofísicos de la Escuela de Ingeniería de Petróleos, especialmente a Luis Felipe Carrillo, Hernando Buendía, Loribeth Atala Buendía y Luz Dary Peña por el apoyo experimental brindado.

A la Universidad Industrial de Santander, Ecopetrol S.A, y Colciencias.

\section{NOMENCLATURA}

$\begin{array}{ll}\mathrm{a}= & \text { Coeficiente de Klein-Nishina. } \\ \mathrm{b}= & 9,8 \times 10^{-24} . \\ \mathrm{CT}= & \text { Tomografía Computarizada. } \\ \mathrm{cp}= & \text { Centipoise. } \\ \mathrm{E}= & \text { Energía Ionizante }[\mathrm{kV}] . \\ \mathrm{f}= & \begin{array}{l}\text { Fracción en peso de cada componente del } \\ \text { fluido. }\end{array} \\ \mathrm{h}= & \text { Espesor }[\mathrm{cm}] . \\ \mathrm{I}= & \begin{array}{l}\text { Intensidad remanente después que los rayos } \\ \mathrm{X} \text { atraviesan el material. }[\mathrm{kV}] .\end{array} \\ \mathrm{I}= & \text { Intensidad del rayo X }[\mathrm{kV}] .\end{array}$




\begin{tabular}{|c|c|}
\hline $\mathrm{kV}=$ & $\begin{array}{l}\text { Energía de los fotones generados en el ánodo } \\
\text { del tubo de rayos } \mathrm{X} \text {. }\end{array}$ \\
\hline $\mathrm{P}=$ & $\begin{array}{l}\text { Razón de electrones de cada elemento res- } \\
\text { pecto al total de electrones que compone una } \\
\text { especie. }\end{array}$ \\
\hline $\mathrm{S}=$ & Fracción volumétrica de determinado fluido. \\
\hline $\mathrm{V}=$ & Volumen de determinado fluido. \\
\hline $\mathrm{wt}=$ & Unidad de concentración peso a peso. \\
\hline$Z=$ & Número atómico. \\
\hline $\mathrm{Z}_{\mathrm{e}}=$ & Número atómico efectivo. \\
\hline$\mu=$ & $\begin{array}{l}\text { Coeficiente de atenuación linear del fluido } \\
\text { que satura completamente el medio poroso. } \\
{\left[\mathrm{cm}^{-1}\right] \text {. }}\end{array}$ \\
\hline$\rho=$ & Densidad $\left[\mathrm{g} / \mathrm{cm}^{3}\right]$. \\
\hline
\end{tabular}

\section{SUBÍNDICES}

$\begin{array}{cl}\mathrm{i}= & \text { Componente del fluido } \\ \mathrm{CO}_{2}= & \text { Dióxido de carbono } \\ \mathrm{g}= & \text { Gas } \\ \mathrm{o}= & \text { Petróleo } \\ \mathrm{T}= & \text { Total } \\ \mathrm{w}= & \text { Agua } \\ \mathrm{wc}= & \text { Agua connata } \\ 1,2= & \text { Energía a la que se realiza la prueba. }\left(E_{1} y E_{2}\right)\end{array}$

\section{CONVERSIÓN DE UNIDADES}

\begin{tabular}{ccc} 
& S.Inglés & SIU \\
Viscosidad & $1 \mathrm{cp}$ & $10^{-3} \mathrm{~Pa}^{*} \mathrm{~s}$ \\
Temperatura & ${ }^{\circ} \mathrm{C}$ & $\left({ }^{\circ} \mathrm{F}-32\right) / 1,8$ \\
\hline
\end{tabular}

\section{REFERENCIAS}

1. Cuñado, C. E., 2012. Estudio geoquímico de los petróleos de los campos Jilguero y Caracara, Cuenca de los Llanos, Colombia. Barcelona: Universidad Politecnica de Catalunya.

2. Dake, L. P., 2005. Fundamentals of Reservoir Engineering. San Diego,USA: Elsevier.

3. Evans, 1955. The Atomic Nucleus. New York City: McGraw-Hill Book Co.
4. Gonçalves Machado, C., 2013. Histerese nas curvas de permeabilidade relativa trifásica em carbonatos usando tomografia computadorizada de raios-X. UNICAMP.

5. Hounsfield, G. N., 1973. Computerized transverse axial scanning (Tomography). Part I. Description of system.. Br. J. Radiol, Volumen 46, pp. 1016-1022.

6. Hove, A. O., Ringen, J. K. \& Read, P. A., 1987. Visualisation of laboratory corefloods with the aid of computerized tomography of X-rays.. SPE, pp. 148-154.

7. Hunt , P. K. \& Engler, P., 1987. Computed tomography as a core analysis tool: applications and artefact reduction techniques. SPE, pp. 197-204.

8. Ledley, R. S., Di Chiro, G., Luessenhop, A. J. \& Twigg, H. L., 1974. Computerized transaxial X-ray tomography of the human body.. Science, Volumen 186, pp. 207-212.

9. Mackay, R. S., 1984. Medical images and displays. Wiley, New York.

10. Payne, J. T. \& McCullough, E. C., 1976. Basic principles of computer-assisted tomography.. Appl. Radiol, pp. 53-60.

11. Peters, E. J. \& Hardham, W. D., 1990. Visualization of fluid displacements in porous media using computed tomography imaging. Journal of petroleum Science and Engineering, Volumen 4, pp. 155-168.

12. Sheng, J. J., 2013. Enhanced Oil Recovery Field Case Studies. Oxford,UK: Elservier.

13. Siddiqui, S., Hicks, P. J. \& Grader, A. S., 1996. Verification of Buckley-Leverett three-phase theory using computerized tomography. Journal of petroleum Science and Engineering, Volumen 15, pp. 1-21.

14. Storm, E. \& Israel, H. I., 1970. Nuclear Data, Tables A7,Photon Cross Sections From $1 \mathrm{Kev}$ to $100 \mathrm{Mev}$ for elements $Z=1$ to $Z=100$. California: University of California. 
15. Vinegar, H. J. \& Wellington, S. L., 1986. Tomographic imaging of three phase flow experiments. American Institute of Physics, 58(1), pp. 95-107.

16. Wang, S. Y., Ayral , S. \& Gryte, C. C., 1984. Computer assited tomography for the obersvation of oil displacement in porous media. SPE, Volumen 4, pp. 53-55.
17. Wellington, S. L. \& Vinegar, H. J., 1987. X-Ray Computerized Tomography. Journal of Petroleum Technology, pp. 885-898.

18. Withjack, E. D., 2003. The Role of X-Ray Computed Tomography in Core Analysis.

19. Withjack, E. M., 1988. Computed tomography for rock-property determination and fluid flow visualization. SPE, pp. 183-196.

Recepción: 7 de agosto de 2016

Aceptación: 18 de octubre de 2016 